\title{
Sincronización de datos en el contexto de procesos terapéuticos de rehabilitación física
}

\section{Synchronization of data in the context of therapeutic physical rehabilitation processes}

\author{
RAMÍREZ-HERNÁNDEZ, Marco Antonio†* \& SANTOS-QUIROZ, Randolfo Alberto \\ Universidad Tecnológica de Xicotepec de Juárez-Área Tecnologías de la Información \\ ID $1^{\mathrm{er}}$ Autor: Marco Antonio, Ramírez-Hernández / ORC ID: 0000-0003-2866-272X \\ ID 1 ${ }^{\mathrm{er}}$ Coautor: Randolfo Alberto, Santos-Quiroz / ORC ID: 0000-0002-6333-4193
}

\section{Resumen}

En el desarrollo de una plataforma de información centrada en las operaciones cotidianas de una clínica de rehabilitación física, actualmente se está trabajando en dos componentes de software principales para su interacción con los usuarios. Una aplicación web centrada en las operaciones del personal laboral (especialistas y profesionistas en formación) y una aplicación móvil nativa enfocada en las acciones de los pacientes, cada una de ellas tienen la necesidad de interactuar con el mismo repositorio de información, analizando el lado del paciente surgen problemáticas de conectividad permanente a los datos principales por medio de Internet o algún otro protocolo de transmisión de datos, surge el requisito de poder interactuar con la información personal generada, mismo que podría lograrse mediante la sincronización de datos entre cliente servidor.

Sincronización de datos, Aplicación Web, Aplicación móvil

\begin{abstract}
In the development of an information platform focused on the daily operations of a physical rehabilitation clinic, two main software components are currently being worked on for their interaction with users. A web application focused on the operations of labor personnel (specialists and training professionals) and a native mobile application focused on the actions of patients, each of them has the need to interact with the same information repository, analyzing the side of the Patient problems arise from permanent connectivity to the main data through the Internet or some other data transmission protocol, the requirement arises to be able to interact with the generated personal information, which could be achieved by synchronizing data between client-server.
\end{abstract}

Data synchronization, Web application, Mobile application 


\section{Objetivo}

Establecer una metodología de comunicación asíncrona entre una base de datos local (particular) y una remota (global empresarial) que involucren un proceso de sincronización recurrente de datos e información, de las operaciones cotidianas de una clínica escuela de rehabilitación física.

\section{Metodología}

En el contexto de las tecnologías de la información, la persistencia es la acción de preservar los datos e información de forma permanente en determinado dispositivo físico de almacenamiento (escribir o guardar), permitiendo en un futuro poder recuperarlos (leer o abrir) para poder procesar, modificar e interpretarlos. El software, que además de interpretar las acciones que el usuario realiza por medio del hardware, es el principal medio de recolección de datos, por lo que las empresas hoy día deciden estructurar, diseñar e implementar aplicaciones informáticas útiles, que se adapten a las necesidades de los usuarios (usabilidad), pero que principalmente les permitan recabar la información respecto a un contexto operativo laboral, cultural o social con el objetivo de contar con la mayor información posible para toma asertiva decisión. Para poder hacer pruebas delimitadas se aplicó al contexto del desarrollo de una app para la construcción del expediente clínico electrónico y gestión de citas médicas para una clínica de rehabilitación física, mismo que permitirá a futuro al paciente interactuar con la información que describe su proceso de rehabilitación, aunque no se esté conectado directamente a la base de datos principal, aunque si con un tiempo límite de sincronización.

\section{Contribución}

La necesidad de sincronizar datos entre dispositivos móviles y servidores es temática que no depende directamente del contexto operacional laboral de un sector productivo o de servicios propios de las empresas o instituciones, debido cómo se comporta el mercado tecnológico la tendencia de la industria 4.0, el uso de las redes informáticas físicas y lógicas, el uso de dispositivos electrónicos personales y la capacidad operativa de ellos, obliga a los desarrolladores de software en pensar como movilizarán los datos generados.
Esta fase de investigación tiene como objetivo diseñar, desarrollar e implementar herramientas tecnológicas que ayuden a la parte administrativa de la clínica escuela de rehabilitación física de la Universidad Tecnológica de Xicotepec de Juárez, institución educativa que proporciona diversas Áreas Académicas. Dentro de las cuales se encuentran Terapia Física Área Rehabilitación, la cual oferta atención a pacientes internos de la Universidad, así como al público en general, por lo que cada día generan una gran cantidad de expedientes clínicos, entre otros documentos importantes para la atención de pacientes.

A futuro mediante la administración de la información generada será posible reducir la deserción de los pacientes a sus procesos de rehabilitación, por el control de procesos derivados de las decisiones tomada por los actores. En este momento el avance del trabajo se encuentra en una fase de construcción de prototipo y simulación de procesos, hay supuestos que por el momento no pueden ser comprobados especialmente los relacionados a la escalabilidad, volumen de información, que una vez se incorporen a la operación de servicios de rehabilitación se podrán evaluar, corregir o adecuar. Los esfuerzos se enfocaron en el diseño, desarrollo y pruebas de una aplicación para la gestión de citas médicas, en la cual un administrador de la clínica, posterior al registro del paciente (Ficha de Identificación) le asignará basado en la disponibilidad una fecha y hora de calendario una cita de valoración, tratamiento (asignación o aplicación), valoración a un paciente y al menos a un personal médico. Dependiendo del tiempo intermedio entre la gestión y la realización, el paciente pudiera presentar situaciones que le obliguen a no asistir a dicha cita, por lo que debería informar a la clínica para que los recursos (humano, material y espacial) reservados puedan ser reasignados.

\section{Introducción}

Esta fase de investigación pertenece es una parte de un proyecto mayor, que tiene como objetivo diseñar, desarrollar e implementar herramientas tecnológicas que ayuden a la parte administrativa de la clínica escuela de rehabilitación física de la Universidad Tecnológica de Xicotepec de Juárez, institución educativa que proporciona diversas Áreas Académicas. 
Dentro de las cuales se encuentran Terapia Física Área Rehabilitación, la cual oferta atención a pacientes internos de la Universidad, así como al público en general, por lo que cada día generan una gran cantidad de expedientes clínicos, entre otros documentos importantes para la atención de pacientes. Las aplicaciones móviles pueden sincronizar datos con los servidores de datos principales por medio de servicios web, que derivarán en la mejora de la usabilidad y experiencia de usuario.

\section{Marco teórico}

Los dispositivos móviles se encuentran por todos lados y en todas las clases sociales, según The Global System Mobile Association (GSMA) a agosto de 2018 existen más de 9,144 millones de conexiones móviles, mientras que el censo de la población según una nota de Excelsior (2018) es de 7,500 millones de personas. Además de que en el año 2014 por primera vez fue superado por primera vez el número y tiempo de acceso a la web desde dispositivos móviles respecto a equipos de escritorio. Esta tendencia está haciendo que las tecnologías móviles estén a la vanguardia.

Un estudio realizado por Bizness Apps reveló que 780 millones de personas son únicamente usuarios móviles. Conforme pasa el tiempo las exigencias de los clientes se ha convertido en el punto principal de referencia para el desarrollo de aplicaciones móviles por lo cual es indispensable realizar programas que sean fáciles de usar para el usuario.

Es por ello el sector empresarial no debe ignorar la interacción que los usuarios tienen con los dispositivos electrónicos personales conocidos como "Smart Phones" o teléfonos inteligentes y los beneficios que le aportarán en la recolección, procesamiento o publicación de datos e información para sus contextos laborales o de mercado. Esto detonará que las empresas enfoquen y refuercen sus proyectos de software a las plataformas móviles, en la actualidad siendo la mayoría para los sistemas operativos Android y IOS.
Con la creciente incorporación de aplicaciones móviles ya sean nativas, web apps o híbridas la interacción entre datos y usuarios interesados, no solo los dueños, es obligatoria, por lo que se requieren de metodologías que aseguren la persistencia y privacidad (Figura 1) de los datos fuera del dispositivo personal, con la finalidad proveer de un mejor producto o servicio. Ubicando a los desarrolladores en pensar una arquitectura de comunicación de datos siendo los servicios web una opción común en la actualidad.

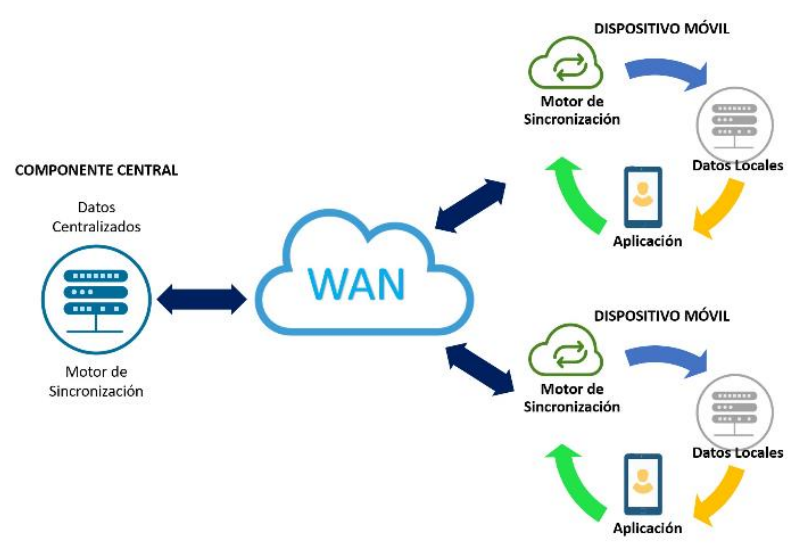

Figura 1 Arquitectura de aplicaciones móviles con motor de sincronización

Un servicio web (en inglés, web service o web services) es una tecnología que utiliza un conjunto de protocolos y estándares que sirven para intercambiar datos entre aplicaciones. Distintas aplicaciones de software desarrolladas en lenguajes de programación diferentes, y ejecutadas sobre cualquier plataforma, pueden utilizar los servicios web para intercambiar datos en redes de ordenadores como Internet.

Los Servicios web en base a su arquitectura, lógica de negocios y apogeo en el mercado y para fines de este estudio los clasificaremos en Servicios SOAP (Simple Object Access Protocol) y REST.

SOAP es un protocolo mucho más formal y estructurado, se centran en la transacción de datos e información, generalmente, con el uso de documentos XML (Extensible Markup Language) lo cual asegura la integridad y robustez, incrementado ligeramente tiempo de procesamiento y complejidad de envoltura (Wrap, mediante un archivo de descripción conocido como WSDL) antes y después de viajar por las carreteras de la nube. 
REST es una tecnología reciente que es más flexible en la transferencia de diversos tipos de datos como XML, JSON, Binarios, también imágenes o documentos de manera más ligera y escalable. Es importante denotar que no existe uno mejor que el otro, ambos tienen ventajas y desventajas que dependen directamente del contexto de aplicación y sus necesidades, REST es recomendable para aplicaciones que funcionan principalmente con el paradigma Modelo Vista Controlador (MVC) de lado del cliente. SOAP con plataformas empresariales más robustas donde la integridad de los datos es incluso legalmente regida.

Aplicando lo anterior al contexto relacionado con la operación de una clínica de rehabilitación, donde, por un lado es necesario la construcción del expediente médico electrónico, la toma de signos vitales, la gestión de citas médicas, la evaluación periódica de los tratamientos, el diagnóstico y pronóstico salubre, los indicadores grupales de conjuntos o sectores de población, entre otras tareas, información que será recolectada y gestionada por una plataforma web de acceso exclusivo para el personal médico.

Además, en algunos casos los pacientes desean cancelar o posponer citas médicas, conocer su situación de salud con términos más coloquiales, incluso gráficos, utilizar medios multimedia para visualizar los ejercicios de rehabilitación que debe realizar de una forma correcta, conocer futuras consecuencias de las patologías o lesiones actuales o pasadas, reflejar la satisfacción del paciente mediante la evaluación de los servicios recibidos, todo será interactuando con una aplicación móvil de acceso personal para los pacientes o sus tutores, lo que refleja la arquitectura de comunicación definida en la Figura 2.

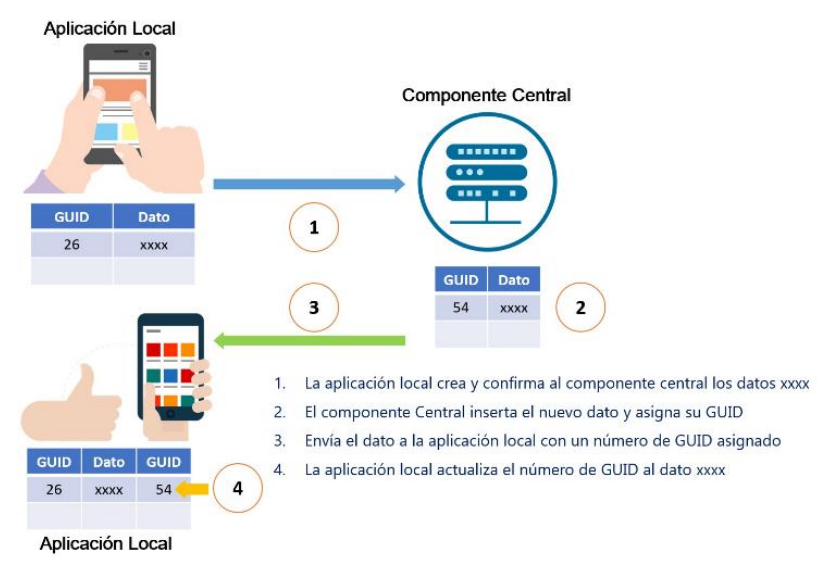

Figura 2 Asignación de GUID a un dato
En cada actividad, proceso o procedimiento que se involucre con lo mencionado genera datos críticos en la toma de decisiones por las partes interesadas, la meta es establecer la sincronía de datos entre ambas aplicaciones mediante el uso de servicios web.

En lo que al contexto específico a la operación de la clínica, el participar e involucrarse con la recopilación de datos sensibles, ha significado un gran reto, que además de requerirse actualizados, disponibles y accesibles, se tiene que pensar en la protección a datos personales íntimos de los pacientes, lo que derivó en conocer a detalle la mecánica de operación de la clínica, la cual se basa en la norma NOM-004-SSA3-2012 del expediente clínico, del cual actualmente se tienen la concentración y resguardo del expediente físico (papel), significando un riesgo considerable a factores como deterioro, ilegibilidad, humedad, perdida o traspapelo.

Para poder comprender el esquema de trabajo y gestión de pacientes, se han llevado a cabo la aplicación de diferentes herramientas administrativas como son los esquemas BPMN (Business Process Model and Notation), previo al diseño y desarrollo del software, derivando en las siguientes fases principales del proceso médico:

- Identificación: Proceso de registro de los datos personales del paciente, para la integración del expediente clínico, así como las cartas responsivas, de consentimiento informado $\mathrm{o}$ de representación para pacientes (menores de edad, no autónomos).

- Valoración: Conjunto de actividades enfocadas a conocer la situación médica y física actual del paciente, incluyendo la toma de signos vitales, síntomas, historial médico o estudios clínicos específicos.

- Diagnóstico: Posterior a la recolección de datos e información, el personal médico, deberá emitir un juicio en relación a la patología actual del paciente. 
- Asignación de Tratamiento: Establecer un conjunto de actividades relacionadas con la mejora de la salud física del paciente, cabe recordar, que el contexto operativo del proyecto de investigación, actualmente solo se enfoca en la medicina física, en el área de rehabilitación. Estas actividades están relacionadas con alternativas de masoterapia, electroterapia, hidroterapia, mecanoterapia, nutrición entre otras, es importante denotar que en esta fase son asignados recursos médicos, tales como médicos generales, terapeutas, sesiones de tratamiento e indicaciones generales.

Aplicación de Tratamiento: Es la parte operativa que el paciente con la atención de los terapeutas y los instrumentos requeridos realizará periódicamente con la finalidad de mejorar su condición física, y que generará los datos necesarios para estadísticamente medir el progreso del paciente y la efectividad del tratamiento, entre otros indicadores significativos del proceso.

Seguimiento del Tratamiento: La duración del tratamiento es variante para cada caso médico, por reglamento se establece un programa de 10 sesiones, posterior a culminarla, se hace una revaloración, con la finalidad de hacer toma de decisiones oportunas.

Alta Médica, en caso de la revaloración del paciente sea favorable o a juicio del personal médico y terapeuta suficiente para suspender el tratamiento, se realizará los registros necesarios.

Análisis de Datos: Esta fase pese a no estar directamente relacionada con el paciente directamente, infiere el uso de los datos de manera conjunta para la obtención de estadísticas salubres de la entidad médica, así como para futuras investigación la posible predicción o recomendación a pacientes.

\section{Resultados}

Los esfuerzos se enfocaron en el diseño, desarrollo y pruebas de una aplicación para la gestión de citas médicas, en la cual un administrador de la clínica, posterior al registro del paciente (Ficha de Identificación) le asignará basado en la disponibilidad una fecha y hora de calendario una cita de valoración, tratamiento (asignación o aplicación), valoración a un paciente y al menos a un personal médico. Dependiendo del tiempo intermedio entre la gestión y la realización, el paciente pudiera presentar situaciones que le obliguen a no asistir a dicha cita, por lo que debería informar a la clínica para que los recursos (humano, material y espacial) reservados puedan ser reasignados.

El proceso de investigación es amplio, por lo es necesario dividir el trabajo en diferente fases tanto de gestión como de operación, la sincronización de datos es importante dado que agregará valor a la herramienta final permitiendo un trabajo fuera de línea, de igual manera es importante estimar la factibilidad de la herramienta, proceso en el cual actualmente se está trabajando, esperando publicar metodologías y resultados al respecto.

\section{Conclusiones}

Al término del proceso se logró desarrollar:

- 7 Servicios Web (Registro Cita, Asignación de Recursos, Solicitud de Cancelación, Sincronización, Recordatorio, Confirmación, Liberación de Recursos).

- $\quad$ Aplicación Web rústica para la gestión de la cita médica por parte del personal de la clínica.

- $\quad$ Aplicación Móvil básica para la gestión de la cita médica por parte del paciente.

- $\quad$ Base de Datos Global para el Servidor de Datos.

- $\quad$ Base de Datos Local para el dispositivo móvil.

Logrando cubrir los siguientes requisitos

- Creación y hospedaje de servicios web en un servidor de aplicación (Glassfish).

- $\quad$ Encriptación de los datos (Una vía con el uso de MD5 y 2 vías con el uso de AES). Creación de bases de datos SQLite en el dispositivo móvil.

RAMÍREZ-HERNÁNDEZ, Marco Antonio \& SANTOSQUIROZ, Randolfo Alberto. Sincronización de datos en el contexto de procesos terapéuticos de rehabilitación física. Revista de Tecnología Informática. 2019 
- Manejabilidad de las herramientas de Android Studio.

- Creación de Recycler Views para mostrar los datos.

- Comunicación entre aplicaciones mediante web services.

- $\quad$ Peticiones HTTP .

- $\quad$ Perseo bidireccional de datos a JSON.

Dejando a futuro o como continuidad al trabajo de investigación

\section{- $\quad$ Pruebas Exhaustivas}

- $\quad$ Usabilidad (UI / UX)

- $\quad$ Diseño Front End (preferentemente con Material Desing)

- Integración a prototipo final.

- Integración a prototipo final.

- Tabla comparativa de resultados REST vs SOAP con datos reales.

Todo lo anterior con la finalidad de sustentar e integrar los datos recopilados durante el proceso de atención médica a los pacientes de la clínica, y construyendo los cimientos para un análisis de datos más profundo y compatible con metodologías de inteligencia artificial y aprendizaje colaborativo a futuro.

\section{Referencias}

A., G. (2016). Mobile Technology Trends . Obtenido de https://www.biznessapps.com/blog/mobiletechnology-trends/

Borda, F. \& Hernández, G. (2006) Una Herramienta para la Sincronización de Datos en Redes Móviles, disponible en : http://sedici.unlp.edu.ar/bitstream/handle/10915 /22443/Documento_completo.PDF?sequence=1 , Consultado: 10 de Julio de 2018

Garrido, J. A. M., \& Domínguez, S. J. A. (2014). La gestión de los sistemas de información en la empresa. Ediciones Pirámide.

Purushothaman, J. (2015). RESTful Java Web Services. Packt Publishing Ltd.
Rodríguez, R. J., Gattini, C., \& Almeida, G. (1999). El establecimiento de sistemas de información en servicios de atención de salud. Guía para el análisis de requisitos, especificación de las aplicaciones y adquisición. Pan American Health Organization. Washington, DC: PAHO.

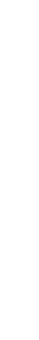

